\title{
Association of $G C K R$ rs780094, alone or in combination with $G C K$ rs1799884, with type 2 diabetes and related traits in a Han Chinese population
}

\author{
Q. Qi • Y. Wu • H. Li • R. J. F. Loos • F. B. Hu • L. Sun • \\ L. Lu • A. Pan • C. Liu • H. Wu • L. Chen - Z. Yu • \\ X. Lin
}

Received: 19 November 2008 / Accepted: 19 January 2009 /Published online: 25 February 2009

(C) Springer-Verlag 2009

\begin{abstract}
Aims/hypothesis The GCKR rs780094 and GCK rs1799884 polymorphisms have been reported to be associated with dyslipidaemia and type 2 diabetes in white Europeans. The aim of this study was to replicate these associations in Han Chinese individuals and to identify the potential mechanisms underlying these associations.

Methods The single nucleotide polymorphisms rs780094 and rs1799884 were genotyped in a population-based sample of Han Chinese individuals $(n=3,210)$ and tested for association with risk of type 2 diabetes and related phenotypes.

Results The GCKR rs780094 A allele was marginally associated with reduced risk of type 2 diabetes (OR 0.85,
\end{abstract}

Q. Qi and Y. Wu contributed equally to this study.

Electronic supplementary material The online version of this article (doi:10.1007/s00125-009-1290-2) contains supplementary material, which is available to authorised users.

Q. Qi $\cdot$ Y. Wu $\cdot$ H. Li $(\varangle) \cdot$ L. Sun $\cdot$ L. Lu $\cdot$ A. Pan $\cdot$ C. Liu $\cdot$

$\mathrm{H}$. Wu $\cdot$ L. Chen $\cdot$ Z. Yu $\cdot$ X. Lin $(\bowtie)$

Key Laboratory of Nutrition and Metabolism, Institute for Nutritional Sciences,

Shanghai Institutes for Biological Sciences,

Chinese Academy of Sciences and Graduate School

of the Chinese Academy of Sciences,

294 Tai-Yuan Road,

Shanghai 200031, People's Republic of China

e-mail: lihx@sibs.ac.cn

e-mail: xlin@sibs.ac.cn

R. J. F. Loos

MRC Epidemiology Unit, Institute of Metabolic Science,

Addenbrooke's Hospital,

Cambridge, UK

F. B. $\mathrm{Hu}$

Department of Nutrition, Harvard School of Public Health,

Boston, MA, USA
95\% CI $0.73-1.00, p$ value under an additive model $\left[p_{(\text {add })}\right]=$ $0.05)$ and significantly associated with reduced risk of impaired fasting glucose (IFG) or type 2 diabetes (OR $0.86,95 \%$ CI $0.77-0.96, p_{[\text {add }]}=0.0032$ ). It was also significantly associated with decreased fasting glucose and increased HOMA of beta cell function (HOMA-B) and fasting triacylglycerol levels $\left(p_{[\mathrm{add}]}=0.0169-5.3 \times 10^{-6}\right)$, but not with HOMA of insulin sensitivity (HOMA-S). The associations with type 2 diabetes and IFG remained significant after adjustment for BMI, while adjustment for HOMA-B abolished the associations. The GCKR rs780094 was also associated with obesity and BMI, independently of its association with type 2 diabetes. The $G C K$ rs 1799884 A allele was significantly associated with decreased HOMA-B $\left(p_{\text {[add }]}=0.0005\right)$, but not with type 2 diabetes or IFG. Individuals with increasing numbers of risk alleles for both variants had significantly lower HOMA-B $\left(p_{[\text {add }]}=5.8 \times 10^{-5}\right)$ in the combined analysis.

Conclusions/interpretation Consistent with observations in white Europeans, the GCKR rs780094 polymorphism contributes to the risk of type 2 diabetes and dyslipidaemia in Han Chinese individuals. In addition, we showed that the effect on type 2 diabetes is probably mediated through impaired beta cell function rather than through obesity.

Keywords Association study · Chinese - GCK · GCKR · Obesity · Type 2 diabetes

$\begin{array}{ll}\text { Abbreviations } \\ \text { CRP } & \text { C-reactive protein } \\ \text { GCK } & \text { Glucokinase } \\ \text { GCKR } & \text { Glucokinase regulatory protein } \\ \text { HOMA-B } & \text { HOMA of beta cell function } \\ \text { HOMA-IR } & \text { HOMA of insulin resistance }\end{array}$


HOMA-S HOMA of insulin sensitivity

IFG Impaired fasting glucose

LD Linkage disequilibrium

NFG Normal fasting glucose

$p_{\text {(add) }} \quad p$ value under an additive model

$p_{\text {(dom) }} \quad p$ value under an dominant model

SNP Single nucleotide polymorphism

\section{Introduction}

Glucokinase (GCK) is the key glucose phosphorylation enzyme responsible for the first rate-limiting step in the glycolysis pathway and regulates glucose-stimulated insulin secretion from pancreatic beta cells and glucose metabolism in the liver [1]. Inactivating $G C K$ mutations lead to maturityonset diabetes of the young and neonatal diabetes [2-4], whereas activating $G C K$ mutations cause persistent hyperinsulinaemic hypoglycaemia [5-8]. Moreover, a common variant $(-30 \mathrm{G}>\mathrm{A}, \mathrm{rs} 1799884)$ in the pancreatic beta cellspecific promoter of $G C K$ has been shown to be associated with increased risk of type 2 diabetes [9], hyperglycaemia [9-11] and impaired beta cell function [9-13].

GCK activity, at least in the liver, is closely regulated by the glucokinase regulatory protein (GCKR) [14]. Animal studies have indicated that $G c k r$ knockout mice show a parallel loss of GCK protein levels and activity in the liver, leading to altered glucose metabolism and impaired glycaemic control [15]. In contrast, mice with excess levels of GCKR show significant improvement of insulin sensitivity and glucose tolerance [16]. Moreover, the genomic region that harbours the GCKR gene has been linked to metabolic traits such as fat mass and circulating leptin concentrations [17]. These observations prompt the hypothesis that genetic variations in GCKR might be diabetogenic or obesogenic if they have functional implications for the synthesis of GCKR that might result in increased inhibitory effect on GCK. This hypothesis is supported by several studies. In the Diabetes Genetics Initiative genome-wide association study for type 2 diabetes and quantitative traits including triacylglycerol levels, GCKR rs780094 A allele was found to be strongly associated with hypertriacylglycerolaemia and also showed a trend toward association with decreased plasma glucose levels, higher insulin sensitivity and a lower risk of type 2 diabetes in populations of European origin [18]. A subsequent replication study in 16,853 Danes confirmed that the rs780094 A allele was associated with increased fasting triacylglycerol, impaired fasting and OGTT-related insulin release, reduced HOMA of insulin resistance (HOMA-IR), increased risk of dyslipidaemia and a modestly decreased risk of type 2 diabetes. An additive effect of $G C K$ rs 1799884 and GCKR rs780094 risk alleles on plasma glucose and insulin release was also observed [19]. Recently, the DESIR prospective cohort study demonstrated that the GCKR variant rs 1260326 (also referred to as $\mathrm{P} 446 \mathrm{~L}$ ), in strong linkage disequilibrium (LD) with rs780094 ( $r^{2}=0.93$ in the HapMap Centre d'Etude du Polymorphisme Humain [Utah Residents with Northern and Western European Ancestry] [CEU] population), displayed similar associations with type 2 diabetes, dyslipidaemia and related phenotypes in a general French population, with more pronounced effects on the same glucose homeostasis variables, including fasting glucose, fasting insulin, HOMA-IR and risk of hyperglycaemia [20], than those observed for GCKR rs780094 in the Finnish, Swedish and Danish populations $[18,19]$. Consistent with this, GCKR variant rs1260326 was previously reported to be associated with increased insulin secretion in 520 Danish twins [21]. The most recent study, combining data from 12 independent cohorts comprising more than 45,000 individuals with various ethnic backgrounds, provided convincing evidence that GCKR rs780094 is strongly associated with different effects on fasting plasma triacylglycerol and glucose concentrations in multiple populations and demonstrated that the non-synonymous rs1260326 variant represents the strongest association signal in the region [22].

The objectives of the present study were twofold: (1) to examine whether the associations previously observed for $G C K$ rs 1799884 and both GCKR rs1260326 and rs780094 variants in white Europeans could be replicated in a general Chinese population including 3,210 unrelated individuals from Beijing and Shanghai; and (2) to explore the potential mechanisms underlying the associations observed in Han Chinese individuals.

\section{Methods}

Study population The study sample consisted of 3,210 individuals (1,423 men, 1,787 women) from the Study on Nutrition and Health of Ageing Population in China, a population-based study among non-institutionalised and unrelated Han Chinese individuals aged 50 to 70 years from Beijing and Shanghai. The study population, design and protocols have been described in detail elsewhere [23]. All participants underwent a complete physical examination including standard anthropometric measurements, overnight fasting blood sample collection and completion of questionnaires on medical history, nutrition and physical activity. Height, weight and waist circumference $(\mathrm{cm})$, as well as fasting glucose, insulin, $\mathrm{HbA}_{1 \mathrm{c}}$, triacylglycerol, HDLcholesterol and $\mathrm{C}$-reactive protein (CRP) were measured following standard protocols as described in previous studies $[23,24]$. BMI was calculated as weight $(\mathrm{kg}) /$ height $^{2}\left(\mathrm{~m}^{2}\right)$. HOMA of insulin sensitivity (HOMA-S) and of beta cell function (HOMA-B) were estimated by the HOMA model using Levy's computer model [25]. The study was approved 
by the Institutional Review Board of the Institute for Nutritional Sciences, with written informed consent from all participants. The phenotypic characteristics of the population are shown in Table 1.

Obesity, diabetes and dyslipidaemia Normal weight, overweight and obesity were defined as BMI $<24,24-28$ and $\geq 28 \mathrm{~kg} / \mathrm{m}^{2}$, respectively, according to the Chinese criteria [26]. Type 2 diabetes was defined by 1999 WHO criteria or previously diagnosed type 2 diabetes. Normal fasting glucose (NFG) and impaired fasting glucose (IFG) were defined as fasting glucose $<5.6 \mathrm{mmol} / 1(100 \mathrm{mg} / \mathrm{dl})$ and 5.6 to $7.0 \mathrm{mmol} / \mathrm{l}(126 \mathrm{mg} / \mathrm{dl})$, respectively. Dyslipidaemia was defined by WHO criteria as triacylglycerol $\geq 1.7 \mathrm{mmol} / \mathrm{l}$, HDL-cholesterol $<0.9$ or $<1.0 \mathrm{mmol} / 1$ for men or women respectively, or self-reported current treatment with lipidlowering medication. Of 3,210 participants, 424 had type 2 diabetes (267 previously diagnosed, 157 screen-detected and treatment-naive), 878 had IFG (all screen-detected and treatment-naive) and 1,908 had NFG.

Genotyping Genomic DNA was extracted from peripheral blood leucocytes by a salting out procedure (http://www. protocol-online.org/prot/Detailed/3171.html, accessed 1 January 2009). Single nucleotide polymorphism (SNP) genotyping was performed with a genotyping system (GenomeLab SNPstream; Beckman Coulter, Fullerton, CA, USA) or sequence detection system (ABI PRISM 7900 HT; Applied Biosystems, Foster City, CA, USA) according to the manufacturer's protocols. We first genotyped GCKR rs780094 and $G C K$ rs1799884, and further genotyped $G C K R$ rs1260326 (in strong LD with rs780094, $r^{2}=0.89$ and 0.82 in our population and the HapMap Han
Chinese in Beijing, China [CHB] sample [http:/www. hapmap.org/cgi-perl/gbrowse/hapmap24_B36/, accessed 1 January 2009], respectively), as it has been reported to be the potential causal variant [20]. The genotyping success rates of $G C K R$ rs1260326, rs780094 and GCK rs1799884 were $98.6,98.8$ and $98.3 \%$, respectively. The concordance rates were $100 \%$ for rs 1260326 and $99.2 \%$ for rs 780094 and rs 1799884 , based on $12 \%$ duplicate samples $(n=384)$. The allele frequencies of all SNPs were comparable with the HapMap CHB database and all in Hardy-Weinberg equilibrium $(p>0.14)$.

Statistical methods Hardy-Weinberg equilibrium was tested using a likelihood ratio test. The ORs and 95\% CIs were estimated by logistic regression under the additive and dominant models, adjusting for sex, age, BMI (where appropriate) and geographical region (Shanghai/Beijing) to determine the association between each SNP and type 2 diabetes, IFG, obesity and overweight. Generalised linear regression was applied to quantitative trait analyses in which participants with known diabetes or receiving glucose-lowering treatment $(n=267)$ were excluded and adjusting for age, sex, geographical region and BMI (where appropriate) under the additive and dominant models. Insulin, HOMA-S, triacylglycerol and CRP were natural log-transformed before analyses and the data presented as geometric means. Gene $\times$ gene interactions were assessed by including the respective interaction terms of SNPs under additive model in logistic regressions using the likelihood ratio test. The combined effects of the SNPs on risk of type 2 diabetes and/or IFG, and on related quantitative traits were evaluated by logistic regression and generalised linear regression, respectively, after categorising the participants
Table 1 Characteristics of the study population

Unless otherwise indicated, data are means $\pm \mathrm{SD}$ or medians (interquartile range)

\begin{tabular}{llll}
\hline Characteristic & All & Men & Women \\
\hline$n(\%)$ & 3,210 & $1,423(44.3)$ & $1,787(55.7)$ \\
Age (years) & $58.6 \pm 6.0$ & $58.8 \pm 5.9$ & $58.4 \pm 6.1$ \\
BMI $\left(\mathrm{kg} / \mathrm{m}^{2}\right)$ & $24.4 \pm 3.6$ & $24.1 \pm 3.3$ & $24.7 \pm 3.8$ \\
Waist circumference (cm) & $83.6 \pm 10.6$ & $85.5 \pm 10.5$ & $82.2 \pm 10.2$ \\
Fasting glucose (mmol/1) & $5.84 \pm 1.74$ & $5.95 \pm 1.89$ & $5.74 \pm 1.59$ \\
HbA 1 (\%) & $5.99 \pm 1.10$ & $5.98 \pm 1.14$ & $6.00 \pm 1.07$ \\
Fasting insulin (pmol/1) & $82.2(59.4-112.2)$ & $74.4(52.8-102.0)$ & $88.2(64.8-119.4)$ \\
HOMA-B & $110.3 \pm 47.0$ & $101.6 \pm 45.6$ & $117.2 \pm 46.9$ \\
HOMA-S & $63.7(47.1-86.9)$ & $69.5(51.1-96.6)$ & $59.3(44.6-81.1)$ \\
Triacylglycerol (mmol/l) & $1.09(0.75-1.67)$ & $1.03(0.70-1.63)$ & $1.13(0.80-1.68)$ \\
HDL-cholesterol (mmol/1) & $1.28 \pm 0.33$ & $1.22 \pm 0.33$ & $1.32 \pm 0.33$ \\
CRP (mg/l) & $0.68(0.33-1.50)$ & $0.67(0.33-1.48)$ & $0.69(0.33-1.53)$ \\
Overweight, $n(\%)$ & $1,224(38.1)$ & $531(37.3)$ & $693(38.8)$ \\
Obesity, $n(\%)$ & $474(14.8)$ & $177(12.4)$ & $297(16.6)$ \\
IFG, $n(\%)$ & $878(27.4)$ & $425(29.9)$ & $453(25.4)$ \\
Type 2 diabetes, $n(\%)$ & $424(13.2)$ & $207(14.6)$ & $217(12.1)$ \\
\hline
\end{tabular}


according to the number of the risk alleles they carried. The data from both geographical regions were pooled for analysis. Similar $p$ values and ORs estimated by metaanalysis suggest pooling does not introduce potential bias in this study (Electronic supplementary material [ESM] Tables 1 and 2). All reported $p$ values are nominal and twosided. Association analyses were performed with SAS version 9.1 (SAS Institute, Cary, NC, USA). Power calculations were performed using Quanto software (http://hydra.usc.edu/gxe/, accessed 1 January 2009).

\section{Results}

Consistent with previous studies, the GCKR rs780094 A allele was highly associated with higher levels of plasma triacylglycerol $\left(p\right.$ value under an additive model $\left[p_{(\text {add })}\right]=5.3 \times 10^{-6}$, $p$ value under an dominant model $\left[p_{(\mathrm{dom})}\right]=1.7 \times 10^{-6}$ ) (Table 3) and increased risk of dyslipidaemia under either an additive (OR $1.15,95 \%$ CI $\left.1.03-1.29, p_{\text {[add] }}=0.011\right)$ or dominant model (OR 1.28, 95\% CI 1.06-1.56, $p_{[\text {dom] }}=0.011$ ). However, we observed no significant associations between plasma CRP levels and GCKR rs780094 $(p=0.56)$ and $G C K$ rs1799884 ( $p=0.42)$, as had been suggested in the previous studies, although the mean values of three genotype groups at rs780094 tended in the same direction as the original studies [22] (Table 3).

We then examined whether the association between GCKR rs780094 A allele and decreased risk of type 2 diabetes also existed in Han Chinese individuals. The GCKR rs780094 A allele was marginally associated with a decreased risk of type 2 diabetes (OR $0.86,95 \%$ CI $0.73-1.01, p_{\text {[add] }}=0.07$ ) under an additive model and significantly associated (OR $0.69,95 \%$ CI $0.53-0.89, p_{[\text {dom] }}=0.0052$ ) under a dominant model. Similarly, we also observed a significant association between the GCKR rs780094 A allele and reduced risk of the combined phenotypes of type 2 diabetes and IFG under both additive (OR 0.84, 95\% CI $\left.0.75-0.94, p_{\text {[add] }}=0.0014\right)$ and dominant genetic models (OR $0.67,95 \%$ CI $0.55-0.89$, $p_{\text {[dom }]}=1.5 \times 10^{-5}$ ).

We next tested for association with obesity and overweight to see whether they are possible mediators of the association between GCKR rs780094 and diabetes. As shown in Table 2, the GCKR rs780094 A allele showed a marginal association with decreased risk of obesity (OR $0.88,95 \%$ CI $0.75-1.02, p_{\text {[add] }}=0.09$ ) and a significant association with decreased risk of combined overweight/ obesity (OR $0.87,95 \%$ CI $\left.0.78-0.96, p_{\text {[add] }}=0.0061\right)$ under an additive model, these associations being both significant under a dominant model $\left(\mathrm{OR}=0.74, p_{\text {[dom] }}=0.022\right.$ for obesity; $\mathrm{OR}=0.79, p_{[\mathrm{dom}]}=0.012$ for combined overweight $/$ obesity). Consistently, the GCKR rs780094 A allele was also associated with decreased BMI $\left(p_{\text {[add] }}=0.0204\right.$, $\left.p_{[\mathrm{dom}]}=0.0025\right)$ and waist circumference $\left(p_{\text {[add] }}=0.0125\right.$, $\left.p_{[\mathrm{dom}]}=0.0011\right)$ in the quantitative trait analyses (Table 3 ). To find out whether the associations between GCKR rs780094 and risk of type 2 diabetes and IFG are mediated through its effect on obesity, we re-checked the associations by further adjusting for BMI. The results showed that adjustment for BMI did not materially change the associations of GCKR rs 780094 with type 2 diabetes and IFG (Table 2).

We further tested for the associations of GCKR rs780094 with diabetes-related quantitative traits, including fasting glucose, fasting insulin, HOMA-B and HOMA-S to examine whether these are potential mediators of the GCKR rs780094diabetes association. The GCKR rs780094 A allele was significantly associated with lower fasting glucose levels $\left(p_{\text {[add }]}=0.0093, p_{\text {[dom] }}=0.0007\right)$ and increased HOMA-B $\left(p_{\text {[add }]}=0.0066, p_{[\text {dom }]}=0.00018\right)$, but was not associated with HOMA-S $(p=0.89)$ and fasting insulin levels $(p=0.67)$. Importantly, the association with type 2 diabetes (OR 0.96 , $95 \%$ CI $\left.0.77-1.19, p_{\text {[add] }}=0.69\right)$ was abolished and that with combined IFG/type 2 diabetes (OR $0.89,95 \%$ CI $0.79-1.01$, $p_{\text {[add] }}=0.06$ ) was also markedly attenuated after further adjustment for the HOMA-B values. To clarify whether impaired beta cell function (estimated as decreased HOMA-B values) could indeed cause type 2 diabetes, we did a Mendelian randomisation analysis (triangulation approaches) by taking $G C K R$ rs780094 as an instrumental variable (ESM Table 3). We found that the expected effect size, estimated by the magnitude of the association between GCKR rs780094 genotypes and HOMA-B values, and of that between the HOMA-B values and type 2 diabetes risk, was similar to the observed effect size of GCKR rs780094 genotypes on type 2 diabetes risk $(p=0.93)$. Furthermore, adjustment for HOMA-B values abolished the association between $G C K R$ rs 780094 and risk of type 2 diabetes $(\beta z 0.04$, $95 \% \mathrm{CI}-0.18$ to $\sim 0.26, p=0.70$ ). These results suggest that the association between HOMA-B values and type 2 diabetes risk is causal and unconfounded, and that the association of GCKR rs780094 with type 2 diabetes is mediated through beta cell function.

To test whether the obesity status modulates the association with type 2 diabetes, we also analysed the association of GCKR rs780094 with type 2 diabetes in different BMI groups. We found no significant association (OR 0.70, 95\% CI 0.36-1.37, $p=0.30$ ) between the GCKR rs780094 variant and type 2 diabetes in the group with BMI $\geq 28 \mathrm{~kg} / \mathrm{m}^{2}$ and marginal association in the groups with BMI $<24 \mathrm{~kg} / \mathrm{m}^{2}$ (OR $0.68,95 \%$ CI $0.46-1.04, p=0.07$ ) or BMI 24 to $28 \mathrm{~kg} / \mathrm{m}^{2}$ (OR $0.68,95 \%$ CI $0.45-1.03, p=0.07$ ) under dominant model ( $p$ for interaction $=0.98$ ). To find out whether BMI mediates the association between GCKR rs780094 and both HOMA-B and fasting glucose, we tested 


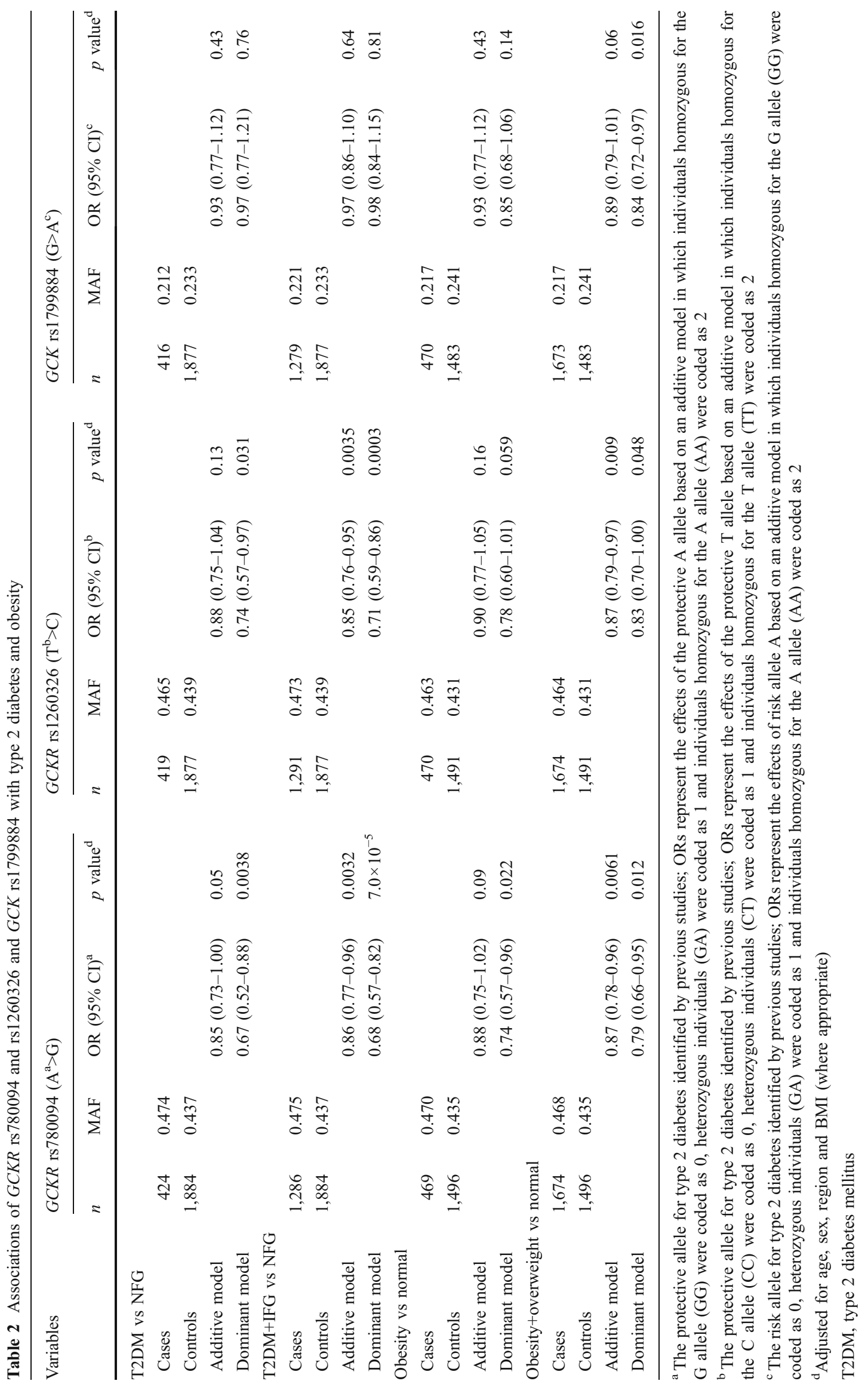


Table 3 Associations of GCKR rs780094 and rs1260326, and GCK rs1799884 with quantitative traits related to type 2 diabetes and obesity in 2,943 treatment-naive participants

\begin{tabular}{|c|c|c|c|c|c|c|c|c|c|}
\hline Variables & $n$ & $\begin{array}{l}\text { Glucose } \\
(\mathrm{mmol} / \mathrm{l})^{\mathrm{a}}\end{array}$ & $\begin{array}{l}\text { Insulin } \\
(\mathrm{pmol} / \mathrm{l})^{\mathrm{a}, \mathrm{b}}\end{array}$ & $\begin{array}{l}\text { HOMA-B } \\
(\%)^{\mathrm{a}}\end{array}$ & $\begin{array}{l}\text { HOMA-S } \\
(\%)^{\mathrm{a}, \mathrm{b}}\end{array}$ & $\begin{array}{l}\text { TG } \\
(\mathrm{mmol} / \mathrm{l})^{\mathrm{a}, \mathrm{b}}\end{array}$ & $\begin{array}{l}\text { BMI } \\
\left(\mathrm{kg} / \mathrm{m}^{2}\right)^{\mathrm{c}}\end{array}$ & $\begin{array}{l}\text { WC } \\
(\mathrm{cm})^{\mathrm{c}}\end{array}$ & $\begin{array}{l}\text { CRP } \\
(\mathrm{mg} / \mathrm{l})^{\mathrm{a}, \mathrm{b}}\end{array}$ \\
\hline \multicolumn{10}{|c|}{ GCKR rs780094 } \\
\hline $\mathrm{AA}$ & 847 & $5.55 \pm 0.04$ & $79.0(1.3)$ & $114.2 \pm 1.5$ & $66.4(1.1)$ & $1.16(0.02)$ & $24.3 \pm 0.1$ & $83.3 \pm 0.4$ & $0.72(0.03)$ \\
\hline $\mathrm{AG}$ & 1,478 & $5.55 \pm 0.03$ & $79.8(1.0)$ & $114.3 \pm 1.1$ & $66.2(0.8)$ & $1.13(0.02)$ & $24.2 \pm 0.1$ & $83.3 \pm 0.3$ & $0.69(0.02)$ \\
\hline GG & 569 & $5.73 \pm 0.05$ & $76.7(1.5)$ & $108.1 \pm 1.8$ & $67.8(1.4)$ & $1.01(0.02)$ & $24.7 \pm 0.1$ & $84.9 \pm 0.4$ & $0.68(0.03)$ \\
\hline$p_{\text {(add) }}$ value & & 0.0168 & 0.31 & 0.0169 & 0.47 & $5.3 \times 10^{-6}$ & 0.0204 & 0.0125 & 0.33 \\
\hline$p_{(\mathrm{dom})}$ value $^{\mathrm{d}}$ & & 0.0017 & 0.11 & 0.0021 & 0.28 & $1.7 \times 10^{-6}$ & 0.0025 & 0.0011 & 0.56 \\
\hline \multicolumn{10}{|c|}{ GCKR rs1260326 } \\
\hline $\mathrm{TT}$ & 867 & $5.55 \pm 0.04$ & $79.6(1.3)$ & $115.0 \pm 1.5$ & $65.9(1.0)$ & $1.17(0.02)$ & $24.3 \pm 0.1$ & $83.4 \pm 0.3$ & $0.72(0.03)$ \\
\hline $\mathrm{TC}$ & 1,460 & $5.55 \pm 0.03$ & $79.5(1.0)$ & $114.3 \pm 1.1$ & $66.1(1.0)$ & $1.13(0.02)$ & $24.3 \pm 0.1$ & $83.4 \pm 0.3$ & $0.69(0.02)$ \\
\hline $\mathrm{CC}$ & 573 & $5.72 \pm 0.05$ & $76.2(1.5)$ & $106.9 \pm 1.7$ & $68.5(1.4)$ & $1.01(0.02)$ & $24.7 \pm 0.1$ & $84.6 \pm 0.4$ & $0.67(0.03)$ \\
\hline$p_{(\text {add })}$ value & & 0.0137 & 0.12 & 0.0011 & 0.17 & $1.3 \times 10^{-6}$ & 0.054 & 0.069 & 0.23 \\
\hline$p_{(\mathrm{dom})}$ value $^{\mathrm{d}}$ & & 0.0025 & 0.058 & 0.0001 & 0.10 & $6.6 \times 10^{-7}$ & 0.017 & 0.015 & 0.33 \\
\hline \multicolumn{10}{|c|}{$G C K$ rs1799884 } \\
\hline GG & 1,711 & $5.56 \pm 0.03$ & $79.8(0.7)$ & $115.7 \pm 1.0$ & $65.8(0.7)$ & $1.13(0.02)$ & $24.4 \pm 0.8$ & $84.0 \pm 0.2$ & $0.69(0.02)$ \\
\hline GA & 1,031 & $5.65 \pm 0.04$ & $78.5(1.2)$ & $109.7 \pm 1.3$ & $66.8(1.0)$ & $1.09(0.08)$ & $24.2 \pm 0.1$ & $83.1 \pm 0.3$ & $0.72(0.02)$ \\
\hline $\mathrm{AA}$ & 151 & $5.48 \pm 0.10$ & $76.5(3.0)$ & $109.7 \pm 3.4$ & $70.1(2.7)$ & $1.13(0.05)$ & $24.5 \pm 0.3$ & $83.8 \pm 0.8$ & $0.65(0.06)$ \\
\hline$p_{(\text {add })}$ value & & 0.42 & 0.23 & 0.0005 & 0.12 & 0.19 & 0.38 & 0.10 & 0.74 \\
\hline$p_{(\mathrm{dom})}$ value $^{\mathrm{d}}$ & & 0.12 & 0.36 & 0.0002 & 0.29 & 0.07 & 0.22 & 0.05 & 0.42 \\
\hline
\end{tabular}

Data are means \pm SE or geometric means (SE) unless otherwise indicated

Participants previously diagnosed with type 2 diabetes or receiving glucose-lowering treatment $(n=267)$ were excluded from the analyses

${ }^{\text {a }}$ Adjusted for age, sex, region and BMI

${ }^{\mathrm{b}}$ Log-transformed before analysis

${ }^{\mathrm{c}}$ Adjusted for age, sex and region

${ }^{\mathrm{d}}$ Dominant model was used for analyses by comparing A allele carriers with GG homozygotes, $\mathrm{T}$ allele carriers with CC homozygotes and A allele carriers with GG homozygotes for GCKR rs780094, GCKR rs1360326 and GCK rs1799884, respectively

TG, triacylglycerol; WC, waist circumference

for associations by further adjusting for BMI. The results showed that the associations of GCKR rs780094 with HOMA-B and fasting glucose remained significant after adjusting for BMI (Table 3). Moreover, the association of $G C K R$ rs 780094 with risk of combined overweight/obesity remained significant after further adjusting for type 2 diabetes (OR $0.88,95 \%$ CI $0.79-0.98, p_{\text {[add] }}=0.015$ ) or HOMA-B (OR $0.85,95 \%$ CI $0.76-0.94, p_{\text {[add] }}=0.002$ ). Therefore, the effects of GCKR rs780094 A allele on type 2 diabetes and obesity seem to be independent in this Chinese population.

As for the GCK rs1799884 polymorphism, its minor A allele exhibited a marginal association with decreased risk of combined overweight and obesity $\left(\mathrm{OR}=0.89, p_{\text {[add] }}=0.06\right.$ and $\mathrm{OR}=0.84, p_{[\mathrm{dom}]}=0.016$, respectively) and a significant association with decreased HOMA-B value $\left(p_{[\text {add }]}=0.0005\right.$, $\left.p_{[\mathrm{dom}]}=0.0002\right)$. No association was seen with type 2 diabetes or IFG. Analyses stratified by sex or region did not change the observations above (data not shown).

We also tested for gene $\times$ gene interaction between $G C K R$ rs780094 and $G C K$ rs1799884 variants in the type 2 diabetes and related traits. Significant interactions were only observed for fasting glucose, with the GCKR rs780094 $\mathrm{G}$ allele being significantly associated with higher fasting plasma glucose in individuals carrying the GA $(p=0.002)$ and AA genotypes ( $p=0.0099$ ), but not in those carrying the GG genotype of $G C K(p=0.73)$ ( $p$ for interaction=0.026; Fig. 1 a). In the analyses of the joint effects, the risk alleles from these two SNPs exhibited a significant combined effect on HOMA-B values, assuming that rs780094 $\mathrm{G}$ allele and rs1799884 A allele were the risk alleles. Participants with increasing numbers of risk alleles had significantly lower HOMA-B values ( $p=5.8 \times 10^{-5}$; Fig. $1 \mathrm{c}$ ).

We further genotyped GCKR rs1260326, as it has previously been reported to be the potential causal variant [20]. As shown (Tables 1, 2 and 3, Fig. 1), the two GCKR variants rs1260326 and rs780094 displayed similar associations with type 2 diabetes, obesity and related phenotypes, as well as similar interaction and combined effects with $G C K$ rs1799884. Therefore, no evidence suggests that GCKR rs1260326 is more informative than GCKR rs780094. 

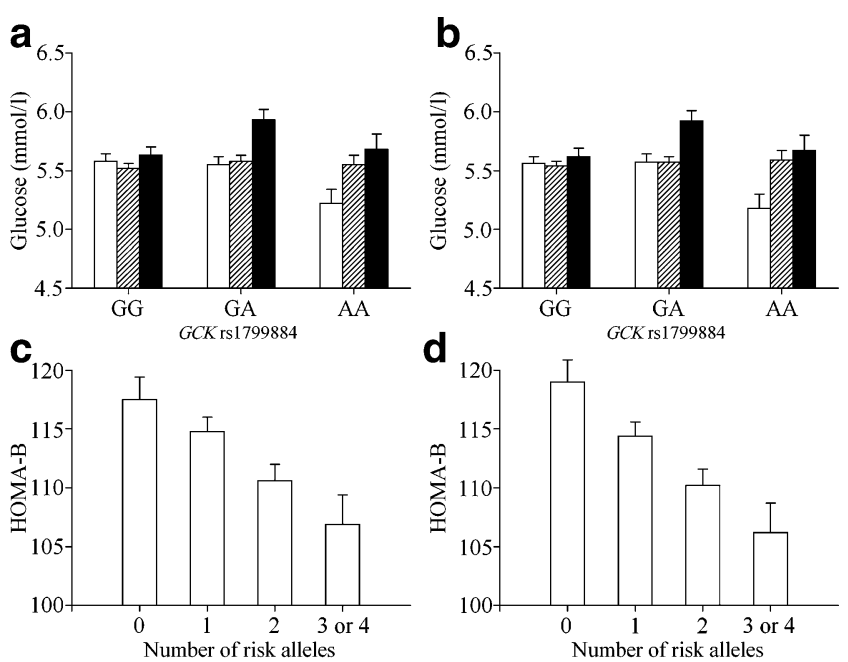

Fig. 1 Gene $\times$ gene interactions for fasting glucose between $G C K R$ rs780094 and $G C K$ rs1799884 (a), and GCKR rs1260326 and $G C K$ rs1799884 (b). White bars, GCKR rs780094 AA and rs1260326 TT genotypes; hatched bars, GCKR rs780094 AG and rs1260326 TC genotypes; black bars, GCKR rs780094 GG and rs1260326 CC genotypes. Additive effects of risk alleles from GCKR rs780094 and $G C K$ rs1799884 SNPs (c), and from GCKR rs1260326 and $G C K$ rs1799884 SNPs (d) on beta cell function estimated by HOMA-B. Risk alleles are the $\mathrm{G}$ allele of $G C K R$ rs780094, the C allele of $G C K R$ rs1260326 and the A allele of $G C K$ rs1799884. Data are geometric means (SE) after adjustment for age, sex, region and BMI. $p=0.026$ and 0.034 for interactions between GCKR rs780094 and $G C K$ rs1799884 SNPs (a), and between GCKR rs1260326 and $G C K$ rs1799884 (b) on fasting glucose, respectively. The GCKR rs780094 $\mathrm{G}$ allele and rs1260326 C allele were significantly associated with higher fasting plasma glucose in carriers of GA genotype ( $p=0.002$ and 0.0004, respectively) and AA genotype ( $p=0.0099$ and 0.0044 , respectively), but not in carriers of the GG genotype of $G C K$ rs 1799884 ( $p=0.73$ and 0.57 , respectively). $p=5.8 \times 10^{-5}$ and $2.1 \times 10^{-6}$ for additive effects of the GCKR rs780094 $\mathrm{G}$ allele and GCK rs1799884 A allele (c), and of the rs1260326 C allele and GCK rs1799884 A allele on HOMA-B values (d), respectively

\section{Discussion}

We observed that the GCKR rs780094 A allele was independently associated with reduced risk of type 2 diabetes and/or IFG and of obesity and/or overweight in Han Chinese individuals. The minor A allele also showed significant association with higher fasting triacylglycerol, lower fasting glucose, increased beta cell function and lower BMI and waist circumference, the most significant associations being with fasting triacylglycerol. However, the $G C K$ rs 1799884 A allele exhibited significant association with impaired beta cell function only, as estimated by HOMA-B. Further analyses of the interaction between the two SNPs and their combined effects indicated that the risk alleles from these two variants exhibited significant additive effects on HOMA-B and participants with increasing numbers of risk alleles had a significantly lower HOMA-B value.
This study confirmed the protective effect against type 2 diabetes conferred by the GCKR rs780094 variant at the expense of higher triacylglycerol levels and dyslipidaemia risk, as suggested by previous studies in other ethnic populations [18-20, 22]. The GCKR rs780094 A allele exhibited significant association with lower fasting glucose and reduced risk of type 2 diabetes or combined IFG and type 2 diabetes by about $15 \%$ for each additional A allele. Conversely, the same A allele was strongly associated with higher fasting triacylglycerol. The mechanism by which the GCKR rs780094 A allele causes dyslipidaemia and protects against type 2 diabetes remains to be determined. Because the frequency of the GCKR rs780094 A allele is substantially higher in Han Chinese individuals (55\%) than in white Europeans (38\%), and the effect size in this study (OR 0.85) is somewhat more pronounced than that observed in Europeans (OR 0.92), the GCKR rs780094 polymorphism might play an even more important role in diabetes susceptibility in Chinese. The populationattributable risk for type 2 diabetes generated by the risk $\mathrm{G}$ allele of GCKR rs780094 variant was estimated to be 14.2 and $10.4 \%$ for this Chinese population and white Europeans in the previous study [19], respectively.

We next aimed to identify the mediating factors, by which the GCKR rs780094 variant is associated with protection against type 2 diabetes. We found that the GCKR rs780094 A allele was significantly associated with increased beta cell function estimated by HOMA-B and that participants with increasing numbers of risk alleles from GCKR rs780094 and GCK rs1799884 had significantly lower HOMA-B values. Importantly, the association of GCKR rs780094 polymorphism with type 2 diabetes was abolished after further adjustment for HOMA-B. The results of Mendelian randomisation suggest that the association between HOMA-B values and type 2 diabetes risk is causal and unconfounded. Therefore, the protective effect of GCKR rs780094 A allele against type 2 diabetes in this Chinese population seems to be mediated through improved beta cell function. This is a somewhat unexpected finding because previous studies in white Europeans have suggested that the effect of GCKR rs780094 variant on diabetes is possibly mediated through improved insulin sensitivity and hepatic glucose metabolism [18-20, 22]. Although it is still questionable whether GCKR is functional in pancreatic beta cells, there is evidence that GCKR is present in pancreatic islets [27]. A variant in GCKR might potentially influence insulin release by altering the regulatory role of GCKR on GCK activity in pancreatic beta cells, if they play a similar role in regulation of GCK activity as in the liver. Therefore, variation in GCKR function and levels of the protein in pancreatic islets between Han Chinese and white individuals may be a possible reason for the difference in the HOMA-B 
association, which remains to be determined. However, it is also possible that because our population is relatively older, the impaired beta cell function might be a more common cause of type 2 diabetes than in the younger populations examined in the previous studies. It has recently been reported that the genetic susceptibility to type 2 diabetes may be modulated by obesity status [28]. To test this hypothesis in Han Chinese individuals, we further stratified analyses for the association between $G C K R$ rs780094 and type 2 diabetes by BMI. Although the associations with type 2 diabetes were marginally significant in non-obese groups and not significant in the obese group under the dominant model, the similar effect size in obese and nonobese groups suggests this difference seems to be due to the smaller sample size in the obese group. Therefore, no evidence was found to suggest that obesity status modulates the association with type 2 diabetes in Han Chinese individuals, but further study with a larger population is warranted to draw a definite conclusion.

Another novel finding is that the GCKR rs780094 A allele was associated with a reduced risk of obesity or overweight and that participants with this protective allele had significantly lower BMI and waist circumference. Moreover, the effects of GCKR rs780094 variant on type 2 diabetes and obesity seem to be independent, because adjustment for BMI did not materially change the results. Consistent with our results, a nominal association between the GCKR rs780094 A allele and lower BMI was observed in an Asian population from the Singapore NHS-98 study $(p=0.04)$ [22]. The mechanisms responsible for the association of GCKR rs780094 with obesity remain to be elucidated. The previous findings that GCK and GCKR are both present in the brain, particularly in the hypothalamus, imply that they might play a similar role in the hypothalamus as in the liver and might be involved in central regulation of energy metabolism [29, 30]. Therefore, the association between GCKR rs780094 and obesity is potentially mediated through its effect on GCK in the hypothalamus. Consistent with this notion, heterozygous $G c k$ knockout mice exhibited increased levels of hypothalamic neuropeptide $\mathrm{Y}$, reduced levels of hypothalamic proopiomelanocortin and elevated food intake [31]. Moreover, the $G C K$ rs1799884 $(-30 \mathrm{G}>\mathrm{A})$ polymorphism has been found to be inversely associated with risk of obesity in Japanese and white Europeans, with carriers of the A allele having a lower incidence of obesity and higher probability of reducing weight $[32,33]$. Our results also showed that $G C K$ rs1799884 A allele was marginally associated with reduced risk of combined obesity and overweight. Another possibility is that GCKR rs780094 is in LD with a nearby variant that is actually associated with obesity in Han Chinese individuals, while in white individuals the LD pattern is different and rs780094 may not in LD with the variant that increases BMI in Asians. This may also explain the discrepancies between our results and those of the previous studies in populations of European origin [18-20]. However, this hypothesis remains to be clarified by fine mapping of the genomic region flanking this variant in the future.

This study also confirms that the $G C K$ rs1799884 risk A allele is strongly associated with impaired beta cell function estimated by HOMA-B in our population, a finding consistent with previous studies [9-13] and with the biological role of GCK in insulin secretion by beta cells [1]. However, we found no association of GCK rs1799884 with type 2 diabetes or related phenotypes, despite the fact that we had minor allele frequencies comparable to those in white individuals and sufficient power (91\%) to detect an OR of 1.34 for type 2 diabetes as the original study suggested [9]. The effect of GCK rs1799884 on beta cell function may not be sufficient to change the risk of type 2 diabetes, an observation consistent with studies in Swedish [34] and Japanese [13, 35] populations. We also failed to find evidence for the previously reported association between GCKR rs780094 and plasma CRP levels [22, $36]$. However, our mean values tended in the same direction as the original studies [22]. Further studies with larger sample sizes are required to provide definite evidence for this association in Han Chinese individuals.

We found significant interactions between GCKR rs780094 and $G C K$ rs1799884 variants on fasting glucose and the GCKR rs780094 $\mathrm{G}$ allele was significantly associated with higher fasting plasma glucose only in the presence of $G C K$ rs 1799884 A allele. Consistently, the risk alleles from these two SNPs exhibited a significant combined effect on HOMA-B values and participants with increasing numbers of risk alleles had significantly lower HOMA-B values. The mechanism underlying the interaction between $G C K R$ rs780094 and GCK rs1799884 variants remains unknown. GCK is a glucose sensor of pancreatic beta cells, which play a crucial role in insulin secretion, and an important regulator of glucose storage and disposal in the liver. Mice lacking hepatic GCK are mildly hyperglycaemic when fasted [37]. We therefore suspect that the $\mathrm{G}$ allele of $G C K R$ rs780094 variant, or a causal variant tagged by it may increase GCKR production, resulting in increased inhibitory activity on GCK in the liver. Consequently, hyperglycaemia may occur in individuals carrying the $G C K$ rs1799884 A allele associated with impaired beta cell function [12]; otherwise normal glucose tolerance would be maintained through insulin secretory compensation in individuals carrying the G alleles of GCK rs1799884 variant. However, this hypothesis remains to be confirmed.

Although GCKR variant rs1260326 was previously identified as the strongest signal in the region [20], we found no evidence here to suggest that rs1260326 is more informative than GCKR rs780094. This is one of the first 
studies examining GCK and GCKR SNPs in relation to type 2 diabetes and related traits in Han Chinese individuals. We therefore chose to report all results without adjustment for multiple testing to highlight all potentially useful associations for subsequent studies and meta-analyses.

In summary, we replicated the association between GCKR rs780094 and type 2 diabetes and dyslipidaemia, as well as the association between GCK rs1799884 and HOMA-B in the Han Chinese population. We also observed additive effects of the GCKR rs780094 and GCK rs1799884 risk alleles on impaired beta cell function estimated as decreased HOMA-B values. Importantly, this is one of the first studies to find the association of GCKR rs780094 polymorphism with obesity and related traits, and the association of $G C K R$ rs780094 A allele with protection against diabetes, which is mediated through improved beta cell function and is independent of that allele's effect on obesity. Therefore, we conclude that GCKR rs780094, either alone or in combination with $G C K$ rs1799884, contributes to the risk of type 2 diabetes, obesity and dyslipidaemia in the Han Chinese population. The effect of GCKR rs780094 polymorphism or a functional variant in LD with it, such as rs1260326, on type 2 diabetes is probably mediated through impaired beta cell function, but not through adiposity.

Acknowledgements This study was funded by research grants from the National Natural Science Foundation of China (30571562), the Knowledge Innovation Program Pilot Project of the Chinese Academy of Sciences (KSCX2-YW-R-73), the National Basic Research Program of China (973 Program 2006CB503902), the Major Projects of Knowledge Innovation Program (KSCX2-YW-R-116 and KSCX1-YW-02) and the Shanghai-Unilever Research Development Fund (CH-2006-0941). We are grateful to all those who participated in the Nutrition and Health of Aging Population in China study. We also thank X. Pang, Z. Zhang, S. Jiao, H. Liu and S. Zou from the Beijing and Shanghai Centers for Disease Control and Prevention for participating in the field work, and our colleagues X. Ye and J. Wang from the Institute for Nutritional Sciences for their part in the laboratory analyses.

Duality of interest The authors declare that there is no duality of interest associated with this manuscript.

\section{References}

1. Matschinsky FM (1996) Banting lecture 1995. A lesson in metabolic regulation inspired by the glucokinase glucose sensor paradigm. Diabetes 45:223-241

2. Froguel P, Zouali H, Vionnet N et al (1993) Familial hyperglycemia due to mutations in glucokinase. Definition of a subtype of diabetes mellitus. N Engl J Med 328:697-702

3. Gloyn AL (2003) Glucokinase (GCK) mutations in hyper- and hypoglycemia: maturity-onset diabetes of the young, permanent neonatal diabetes, and hyperinsulinemia of infancy. Hum Mutat 22:353-362

4. Njolstad PR, Sovik O, Cuesta-Munoz A et al (2001) Neonatal diabetes mellitus due to complete glucokinase deficiency. N Engl J Med 344:1588-1592
5. Christesen HB, Jacobsen BB, Odili S et al (2002) The second activating glucokinase mutation (A456V): implications for glucose homeostasis and diabetes therapy. Diabetes 51:1240-1246

6. Cuesta-Munoz AL, Huopio H, Otonkoski T et al (2004) Severe persistent hyperinsulinemic hypoglycemia due to a de novo glucokinase mutation. Diabetes 53:2164-2168

7. Glaser B, Kesavan P, Heyman M et al (1998) Familial hyperinsulinism caused by an activating glucokinase mutation. N Engl J Med 338:226-230

8. Gloyn AL, Noordam K, Willemsen MA et al (2003) Insights into the biochemical and genetic basis of glucokinase activation from naturally occurring hypoglycemia mutations. Diabetes 52:2433-2440

9. Vaxillaire M, Veslot J, Dina C et al (2008) Impact of common type 2 diabetes risk polymorphisms in the DESIR prospective study. Diabetes 57:244-254

10. Marz W, Nauck M, Hoffmann MM et al (2004) G(-30)A polymorphism in the pancreatic promoter of the glucokinase gene associated with angiographic coronary artery disease and type 2 diabetes mellitus. Circulation 109:2844-2849

11. Rose CS, Ek J, Urhammer SA et al (2005) A -30G $>$ A polymorphism of the beta-cell-specific glucokinase promoter associates with hyperglycemia in the general population of whites. Diabetes 54:3026-3031

12. Stone LM, Kahn SE, Fujimoto WY, Deeb SS, Porte D Jr (1996) A variation at position -30 of the beta-cell glucokinase gene promoter is associated with reduced beta-cell function in middleaged Japanese-American men. Diabetes 45:422-428

13. Yamada K, Yuan X, Ishiyama S et al (1997) Clinical characteristics of Japanese men with glucokinase gene beta-cell promoter variant. Diabetes Care 20:1159-1161

14. Chu CA, Fujimoto Y, Igawa K et al (2004) Rapid translocation of hepatic glucokinase in response to intraduodenal glucose infusion and changes in plasma glucose and insulin in conscious rats. Am J Physiol Gastrointest Liver Physiol 286:G627-G634

15. Farrelly D, Brown KS, Tieman A et al (1999) Mice mutant for glucokinase regulatory protein exhibit decreased liver glucokinase: a sequestration mechanism in metabolic regulation. Proc Natl Acad Sci U S A 96:14511-14516

16. Slosberg ED, Desai UJ, Fanelli B et al (2001) Treatment of type 2 diabetes by adenoviral-mediated overexpression of the glucokinase regulatory protein. Diabetes 50:1813-1820

17. Comuzzie AG, Hixson JE, Almasy L et al (1997) A major quantitative trait locus determining serum leptin levels and fat mass is located on human chromosome 2. Nat Genet 15:273-276

18. Saxena R, Voight BF, Lyssenko V et al (2007) Genome-wide association analysis identifies loci for type 2 diabetes and triglyceride levels. Science 316:1331-1336

19. Sparso T, Andersen G, Nielsen T et al (2008) The GCKR rs780094 polymorphism is associated with elevated fasting serum triacylglycerol, reduced fasting and OGTT-related insulinaemia, and reduced risk of type 2 diabetes. Diabetologia 51:70-75

20. Vaxillaire M, Cavalcanti-Proenca C, Dechaume A et al (2008) The common $\mathrm{P} 446 \mathrm{~L}$ polymorphism in GCKR inversely modulates fasting glucose and triglyceride levels and reduces type 2 diabetes risk in the DESIR prospective general French population. Diabetes 57:2253-2257

21. Koster B, Fenger M, Poulsen P, Vaag A, Bentzen J (2005) Novel polymorphisms in the GCKR gene and their influence on glucose and insulin levels in a Danish twin population. Diabet Med 22:1677-1682

22. Orho-Melander M, Melander O, Guiducci C et al (2008) Common missense variant in the glucokinase regulatory protein gene is associated with increased plasma triglyceride and C-reactive protein but lower fasting glucose concentrations. Diabetes 57:3112-3121

23. Ye X, Yu Z, Li H, Franco OH, Liu Y, Lin X (2007) Distributions of $\mathrm{C}$-reactive protein and its association with metabolic syndrome in middle-aged and older Chinese people. J Am Coll Cardiol 49:1798-1805 
24. Wu Y, Li H, Loos RJ et al (2008) Common variants in CDKAL1, $C D K N 2 A / B, I G F 2 B P 2, S L C 30 A 8$, and HHEX/IDE genes are associated with type 2 diabetes and impaired fasting glucose in a Chinese Han population. Diabetes 57:2834-2842

25. Levy JC, Matthews DR, Hermans MP (1998) Correct homeostasis model assessment (HOMA) evaluation uses the computer program. Diabetes Care 21:2191-2192

26. Bei-Fan Z (2002) Predictive values of body mass index and waist circumference for risk factors of certain related diseases in Chinese adults: study on optimal cut-off points of body mass index and waist circumference in Chinese adults. Asia Pac J Clin Nutr 11(Suppl 8):S685-S693

27. O'Doherty RM, Lehman DL, Telemaque-Potts S, Newgard CB (1999) Metabolic impact of glucokinase overexpression in liver: lowering of blood glucose in fed rats is accompanied by hyperlipidaemia. Diabetes 48:2022-2027

28. Cauchi S, Nead KT, Choquet H et al (2008) The genetic susceptibility to type 2 diabetes may be modulated by obesity status: implications for association studies. BMC Med Genet 9:45

29. Alvarez E, Roncero I, Chowen JA, Vazquez P, Blazquez E (2002) Evidence that glucokinase regulatory protein is expressed and interacts with glucokinase in rat brain. J Neurochem 80:45-53

30. Lynch RM, Tompkins LS, Brooks HL, Dunn-Meynell AA, Levin BE (2000) Localization of glucokinase gene expression in the rat brain. Diabetes 49:693-700
31. Yang XJ, Mastaitis J, Mizuno T, Mobbs CV (2007) Glucokinase regulates reproductive function, glucocorticoid secretion, food intake, and hypothalamic gene expression. Endocrinology 148:1928-1932

32. Gomez-Zumaquero JM, Rojo-Martinez G, Garcia-Escobar E et al (2008) The $-30 \mathrm{G}>\mathrm{A}$ polymorphism of the glucokinase gene promoter is associated with obesity in a population from southern Spain. Obesity (Silver Spring) 16:1973-1975

33. Yamada Y, Kato K, Kameyama T et al (2006) Genetic factors for obesity. Int J Mol Med 18:843-851

34. Lotfi K, Sund G, Lowe R et al (1997) The beta cell glucokinase promoter variant is an unlikely risk factor for diabetes mellitus. Diabetes Incidence Study in Sweden (DISS). Diabetologia 40:959-962

35. Shimokawa K, Sakura H, Otabe S et al (1994) Analysis of the glucokinase gene promoter in Japanese subjects with noninsulindependent diabetes mellitus. J Clin Endocrinol Metab 79:883-886

36. Ridker PM, Pare G, Parker A et al (2008) Loci related to metabolic-syndrome pathways including LEPR, HNF1A, IL6R, and $G C K R$ associate with plasma C-reactive protein: the Women's Genome Health Study. Am J Hum Genet 82:1185-1192

37. Postic C, Shiota M, Niswender KD et al (1999) Dual roles for glucokinase in glucose homeostasis as determined by liver and pancreatic beta cell-specific gene knock-outs using Cre recombinase. J Biol Chem 274:305-315 